\title{
Limit and Shakedown Analysis of Circular Tube Containing External Pit
}

\author{
Xianfeng Wang ${ }^{*}, 1$, Feng Xing ${ }^{1}$, Mingde Xue ${ }^{2}$ and Bingye $\mathrm{Xu}^{2}$ \\ ${ }^{1}$ College of Civil Engineering, Shenzhen University, Shenzhen 518060, PR China \\ ${ }^{2}$ Department of Engineering Mechanics Tsinghua University, Beijing 100081, PR China
}

\begin{abstract}
The circular tubes containing external pit defects are analyzed in a lower-bound finite element computational form based on the static shakedown theorem. The shakedown analysis has not been commonly used in the engineering due to the large amount of computations. To overcome the numerical difficulties, a temperature parameter method is used, in which a pseudo-temperature field is applied to the structure and the resulting self-equilibrium thermoelastic stress is treated as the residual stress field which is used in the analysis. The pseudo temperature is assumed as a harmonic function satisfying the uniqueness theorem, therefore the nodal temperature matrix of the whole structure can be expressed by the boundary nodal temperature matrix. The nonlinear yield condition is piece-wise linearized so that the shakedown analysis is transformed into a linear programming problem in which the strategic variable is boundary nodal temperature and objective variable is the loading multiplier. The relations of limit and shakedown pressures to geometric parameters of various defects are presented.
\end{abstract}

Keywords: Shakedown analysis, thermo elastic stress, residual stress, circular tube, mathematical programming.

\section{INTRODUCTION}

In assessing the load carrying capacity of structures, besides fracture mechanics method which is used for assessing controlling crack, limit and shakedown methods are commonly used for assessing the overall structure collapse, in which limit analysis is for monotonous load, while for the structures subjected to multiple and variable load, shakedown analysis should be required. A combination of the two assessment method by using interpolation between fracture and plastic collapse is called the twocriteria method [1]. In the early time, main concern is paid for the limit analysis using theoretical method. For circular tube with a rectangular slot part through the thickness Kitching and Zarrabi [2] presented experimental limit pressure and lower bound limit analysis [3] based on the assumption of stress resultants in thin shell. The analysis in [3] is similar to that for a circular tube with a rectangular part through slot. No account has been taken of the fact that the mid-surface of the shell in the regions of the slot is offset from the mid-surface of the surrounding thicker shell and that the stress field in the regions of slots and near the slot could not be similar to the stress in thin shell. In the review by Miller [4] a number of approximate results of limit pressure for cylindrical and spherical shells containing defects are reported. All results were based on 2-D or thin shell analysis, however the stress characteristic in a circular tube with defect is a real 3-dimensional problem.

As to plastic limit and shakedown analysis of structure, although the classical shakedown theorem founded by Melan [5] and Koiter [6] and recently developed by Konig [7] is elegant, it is computationally very difficult in applying to 3 -

*Address correspondence to this author at the College of Civil Engineering, Shenzhen University, Shenzhen 518060, P.R. China; Tel: +86-75526534712(office); Fax: +86-755-26534021; E-mail: xfw@szu.edu.cn
D structures due to the large number of variables and constraints. Feng et al., [8-10] investigated a global/local shakedown computational form for three-dimensional elastoplastic strain-hardening and damage structures with cracks. Liu and Cen [11,12] presented a upper bound numerical method for limit analysis of 3-D structures and investigated the upper bound limit analysis of cylindrical shells with part through slots. Later, Konig [13] discussed the exactness of the kinematical approach in the structural shakedown and limit analysis, in which appropriately defined parameters called generalized stresses and strains are used in the analysis.

Comparing with upper bound limit analysis based on displacement variables, lower one on stress has more variables and restraints so that it is computationally more difficult. Fuschi and Polizzotto [14] presented a shakedown load boundary for an elastic-perfectly plastic structure. Giambanco [15] considered the optimal shakedown design of structures discretized by elastic perfectly plastic finite elements. Four alternative methods were presented in his paper to formulate the design problem. Polizzotto [16] provided a unified approach to quasi-static shakedown problems for elastic-plastic solids with piecewise linear yield surface. A general inequality was given by using a perturbation method, from which, by simply specializing the perturbing terms, the generalized Melan theorem as well as bounds on various deformation parameters (such as displacements or plastic strain intensities) were obtained.

The lower bound shakedown analysis of axisymmetric structures is presented by the authors [17] and the limit and shakedown loading for spherical shells containing part through slots and gas holes $[18]$ is investigated. In $[17,18]$ a pseudo-temperature field is put into a structure discretized by finite element method and the resulting thermo-elastic stress is considered as a self-equilibrated stress field. The yield condition is linearized piecemeal. Then the shakedown analysis is transformed into a linear programming containing 
unknown element temperature variables and constraints. By means of the further steps the numerical difficulties for plastic shakedown analysis of 3-D structures are overcome and the results are verified by experiments and by upper bound solution.

\section{DERIVATION OF COMPUTATIONAL FORM}

Static shakedown theorem by Melan [6] is presented as following mathematical programming form

Max: $\mu$

$$
\begin{aligned}
& \text { s.t. } f\left(\mu \sigma_{i j}^{e}\left(x_{k}\right)+\rho_{i j}\left(x_{k}\right)\right) \leq \sigma_{\mathrm{s}} \quad \forall x_{k} \in v \\
& \rho_{i j, j}=0 \quad \forall x_{k} \in v \\
& \rho_{i j} n_{j}=0 \quad \forall x_{k} \in S_{F}
\end{aligned}
$$

where $\sigma_{i j}^{e}$ is the elastic stress field, $\rho_{i j}$ is the self-equilibrate residual stress field, $f$ is the yielding function and $\mu$ is the load multiplier, $v$ and SF represent the structure body and its surface, respectively. The difficulty in lower bound shakedown analysis is to find $\rho_{i j}\left(x_{k}\right)$, which has six independent components at each point $x_{k}$ in a 3-D structure. Since a thermoelastic stress field automatically satisfy self-equilibrate conditions (1b) and (1c) so that a temperature (scalar) field $\theta$ $\left(x_{k}\right)$, instead of the two-order stress tensor fields $\rho_{i j}\left(x_{k}\right)$, can be considered as the optimization variables of the programming problem (1). The procedure may largely simply the programming analysis.

Suppose that the structure which is discretized by finite element method, has $M$ nodes. A pseudo node temperature matrix $\boldsymbol{\theta}$ is applied to the structure. Thus the node thermal stress matrix $\boldsymbol{\rho}$ in the structure has the linear relation with $\boldsymbol{\theta}$ as follows:

$\rho=W \theta$

where $\boldsymbol{W}$ is $(6 \mathrm{M} \times \mathrm{M})$ thermo-elastic matrix of the structure. The yielding function is simulated by an inscribed polyhedron consisting of $\mathrm{J}$ planes as shown in $[4,5]$ :

$\boldsymbol{N}_{j} \boldsymbol{Q}=k_{j} \quad j=1,2 \ldots \mathrm{J}$

where $\boldsymbol{N}_{j}$ is the normal vector to the linearized plane in the stress space and $k_{j}$ is the perpendicular distance from point $\mathrm{O}$ to the $\mathrm{j}$-th linearized plane. $\boldsymbol{Q}$ is the stress of current node, including both elastic stress, $\boldsymbol{Q}^{\boldsymbol{e}}$ and pseudo-residual stress:

$\boldsymbol{Q}=\mu \boldsymbol{Q}^{\boldsymbol{e}}+\boldsymbol{W} \boldsymbol{\theta}$

Therefore the programming problem (1) is transformed into the following problem:

$\operatorname{Max}: \mu$

s.t. $\mu N Q^{e}+N W \boldsymbol{N} \leq \boldsymbol{K}$

where $\mathrm{N}$ is composed of $\mathrm{Nj}$, and $\mathrm{K}$ is a vector composed of kj of all nodes. Problem (5) contains $(\mathrm{M}+1)$ variables including both $\mu$ and node temperature matrix and $(\mathrm{M} \times \mathrm{J})$ constraints, so that the scale of the linear programming usually dependent on the number of nodes of discretized structure. For a 3-D solid finite element analysis the scale of the programming is still too large.

In order to overcome the above mentioned difficulty, the equation for sustained heat conduction without a heat source in the structure is considered. Suppose that there are total M nodes in the structure in which the number of boundary nodes is $\mathrm{B}$, the heat conduction equation in finite element form can be expressed as:

$\boldsymbol{K}^{\boldsymbol{\theta}} \boldsymbol{\theta}=\mathbf{0}$

where $\boldsymbol{K}^{\boldsymbol{\theta}}$ is a $(\mathrm{M} \times \mathrm{M})$ heat conduction matrix and $\boldsymbol{\theta},(\mathrm{M} \times 1)$ node pseudo-temperature matrix. Suppose that the submatrix of the temperature at the boundary nodes, $\boldsymbol{\theta}_{\boldsymbol{b}}$, has been known and $\boldsymbol{\theta}_{\boldsymbol{i}}$ is the sub-matrix of temperature at the nodes inner the structure, then equation (6) is transformed into:

$\left\{\begin{array}{ll}\mathbf{K}_{i i}^{(\theta)} & \mathbf{K}_{i b}^{(\theta)} \\ \mathbf{K}_{b i}^{(\theta)} & \mathbf{K}_{b b}^{(\theta)}\end{array}\right\}\left\{\begin{array}{l}\boldsymbol{\theta}_{i} \\ \boldsymbol{\theta}_{b}\end{array}\right\}=\mathbf{0}$

Therefore, we have

$\theta_{i}=-\boldsymbol{K}_{i i}^{(\theta)-1} \boldsymbol{K}_{i b}^{(\theta)} \theta_{b}$

Thus, $\boldsymbol{\theta}$, can be expressed by

$\theta=\boldsymbol{T} \theta_{b}$

where $\boldsymbol{T}$ is a $(\mathrm{M} \times \mathrm{B})$ matrix:

$\mathbf{T}=\left\{\begin{array}{c}-\mathbf{K}_{i i}^{(\theta)-1} \mathbf{K}_{i b}^{(\theta)} \\ \mathbf{I}\end{array}\right\}$

where I is the identity matrix. Substituting equation (9) into problem (5), the programming problem is transformed into:

Max: $\mu$

s.t. $\mu N Q^{e}+N W T \theta_{b} \leq K$

In programming problem (11) the number of optimization variables is reduced from $(\mathrm{M}+1)$ to $(\mathrm{B}+1)$. The dual programming of problem (11) is solved directly as follows:

Min: $\mu=\boldsymbol{K}^{T} \boldsymbol{Y}$

s.t. $\left(\boldsymbol{N} \boldsymbol{Q}^{e}\right)^{\mathrm{T}} \boldsymbol{Y}=1$

$(N W T)^{\mathrm{T}} Y=0$

$Y \geq 0$

Problem (12) is a standard linear programming form containing $(\mathrm{M} \times \mathrm{J})$ variables $\boldsymbol{Y}$ and $(\mathrm{B}+1)$ constraints. The problem is solved by using Fortran program coded by the authors.

\section{THE LIMIT AND SHAKEDOWN ANALYSIS OF CIRCULAR TUBE WITH PART THROUGH PIT}

The tubes containing four kinds of part-through pit, which are spherical, ellipsoidal, circular and rectangular, on the outside surface shown in Fig. (1) and named for types A, $\mathrm{B}, \mathrm{C}$ and $\mathrm{D}$, respectively, are computed by the presented approach. It is simplified by assuming the two symmetrical planes of each defect to be in the longitudinal and circumferential directions of the cylinder and the region of the defect to be far from the two end of the shell. Therefore, the computing model could be taken a quadrant of the cylinder shown in Fig. (2).

The models are discretized by 3-D 8-node Wilson incompatible element [19] with nine additional degrees of freedom which is accurate to 20-node isoparametric element but the number of degree of freedom decreased by $3 / 5$ (Fig. 3). 

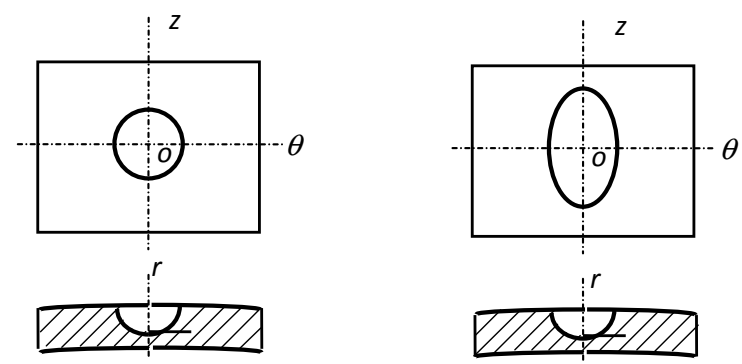

Type A

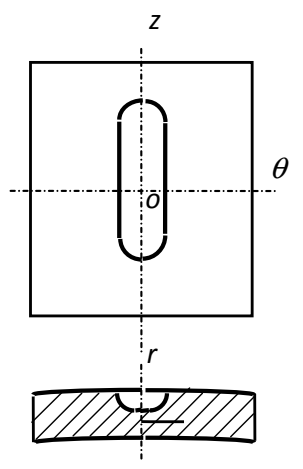

Type C

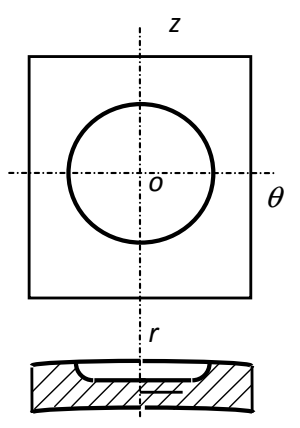

Type D

Fig. (1). Four types of defects.

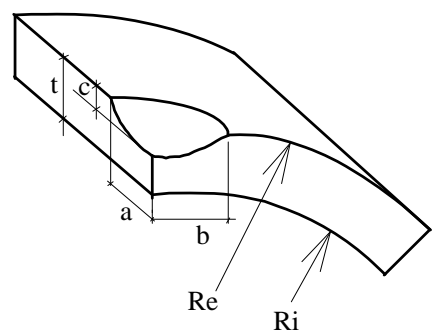

Fig. (2). Computational model.

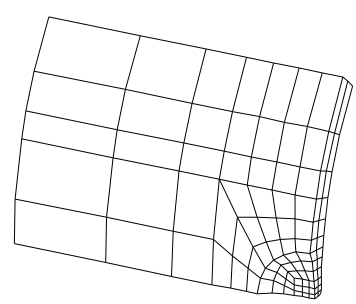

Type A

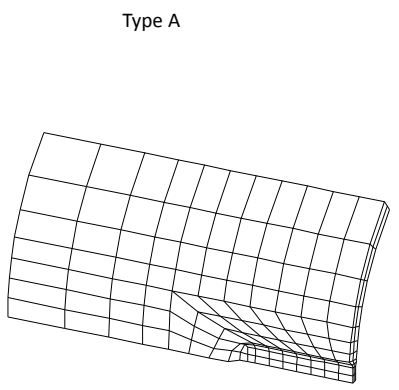

Type C

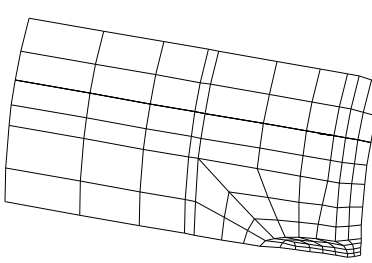

Type B

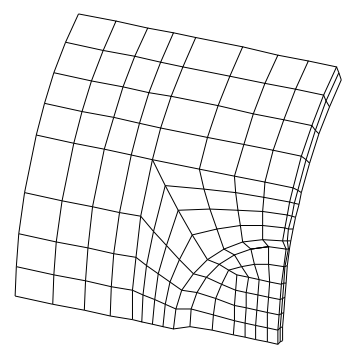

Tvpe D
As to the linearization of the yield condition, as the difference between three principal stresses and stress components $\sigma_{\theta}, \sigma_{z}$ and $\sigma_{r}$ is not significant, $\theta, z$ and $r$ are assumed to be principal axis of stress and Tresca yield condition is chosen as linearized yield condition.

Six steel models are tested and calculated by the presented method. The outer diameter of the tested cylinders are $140 \mathrm{~mm}$ and the yielding and ultimate strength of material: $\sigma_{s}=400 \mathrm{Mpa}, \sigma_{b}=620 \mathrm{Mpa}$. The basic parameters of six models are shown of in Table 1, where $a$ and $b$ are longitudinal and circumferential dimension of slot, respectively, $c$, the depth of the slot and $t$, the thickness of cylinder. According to work criterion for choice of the deformation parameter [20] for each model the experimental limit pressure $p_{l}^{(e x)}$ is obtained from diagram pressure $p /$ volume change $(\Delta v / v)$ and is that for which the permanent volume change is twice the volume change at the initial departure from linearity. This definition corresponds to the definition of ASME code. However, for No1,3,6 models having large areas of defect the definition of ASME code is not appropriate because the volume change at the initial departure from linearity is too large $(\Delta v / v$ large than $1.5 \%)$ to find the limit pressure according to the definition of ASME code. Therefore for the three models the limit pressure is that giving $0.2 \%$ permanent volume change. The tested burst pressure $p_{b}^{(e x)}$ for the experimental models is measured too. The comparison between experimental and calculated results for the six models are given in Table 2. Here, the limit pressure of each test model, $p_{o}$, is calculated with the thickness of each model as follow:

$$
p_{o}=\sigma_{s} \ln \left(R_{o} / R_{i}\right)
$$

\section{Table 1. Basic Parameters of Six Tested Models}

\begin{tabular}{|c|c|c|c|c|c|}
\hline No. & Defect Type & $\mathbf{a}(\mathbf{m m})$ & $\mathbf{b}(\mathbf{m m})$ & $\mathbf{c}(\mathbf{m m})$ & $\mathbf{t}(\mathbf{m m})$ \\
\hline \hline 1 & C & 43.2 & 8.2 & 2.8 & 5.1 \\
\hline 2 & B & 22.2 & 7.4 & 2.9 & 5.4 \\
\hline 3 & D & 26.5 & 26.5 & 2.9 & 5.3 \\
\hline 4 & A & 11.6 & 11.6 & 2.9 & 5.8 \\
\hline 5 & B & 14.9 & 2.6 & 2.5 & 5.1 \\
\hline 6 & C & 89.0 & 8.4 & 2.7 & 5.4 \\
\hline
\end{tabular}

The calculated limit and shakedown pressure, $p_{l}^{(l w)} / p_{o}$ and $p_{s d}^{(l w)} / p_{o}$ by the present method are given in Table 2. The upper bound solution, $p_{l}^{(u p)} / p_{o}$, for the six models is calculated by Liu with the method given in [11] and shown in Table $\mathbf{2}$ as well.

Table 2 shows that:

Fig. (3). Finite element meshes.

The presented results are lower than the upper bound solution for all models but they are closed each other.

The presented results are in good agreement with and lower than the experimental ones. 
Table 2. The Comparison between Numerical and Experimental Results

\begin{tabular}{|c|c|c|c|c|c|c|}
\hline \multirow{2}{*}{ No } & \multicolumn{3}{|c|}{ Tested Results } & \multicolumn{3}{|c|}{ Numerical Results } \\
\hline & $p_{b}^{(e x)}$ (MPa) & $p_{l}^{(e x)}$ (MPa) & $p_{l}^{(e x)} / p_{o}$ & $p_{l}^{(l w)} / p_{o}$ & $p_{l}^{(u p)} / p_{o}$ & $p_{s d}^{(l w)} / p_{o}$ \\
\hline 1 & 26.0 & 22.4 & 0.74 & 0.65 & 0.69 & 0.44 \\
\hline 2 & 28.0 & 24.1 & 0.73 & 0.72 & 0.73 & 0.52 \\
\hline 3 & 28.0 & 23.2 & 0.74 & 0.74 & 0.78 & 0.69 \\
\hline 4 & 40.5 & 32.3 & 0.93 & 0.90 & 0.92 & 0.86 \\
\hline 5 & 30.0 & 27.3 & 0.90 & 0.83 & 0.90 & 0.43 \\
\hline 6 & 23.5 & 17.9 & 0.56 & 0.54 & 0.59 & 0.40 \\
\hline
\end{tabular}

\section{THE EFFECT OF PARAMETERS OF THE DEFECT} ON THE CARRYING CAPACITY OF THE TUBES

The lower bound limit and shakedown pressure of cylinders with four kinds of part-though pits having different parameters are obtained by the present method and given in Figs. (4) (7) which are for Type A, B, C and D, respectively.

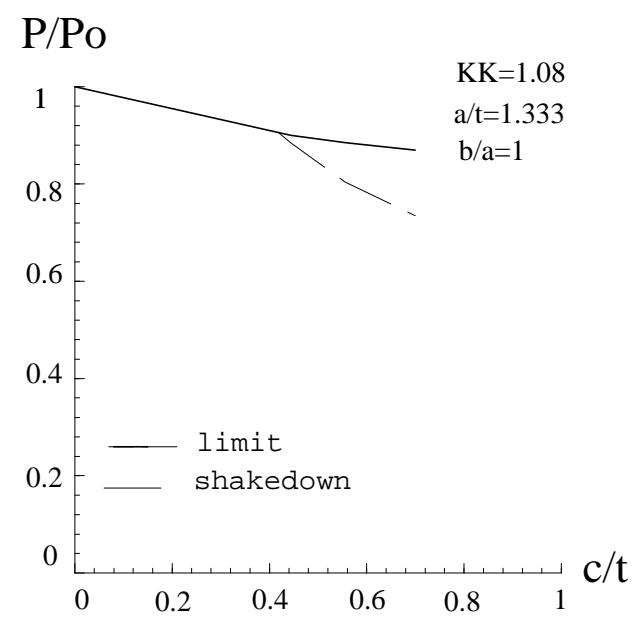

Fig. (4). Effect of depth of type A part-through pit on the carrying capacity.

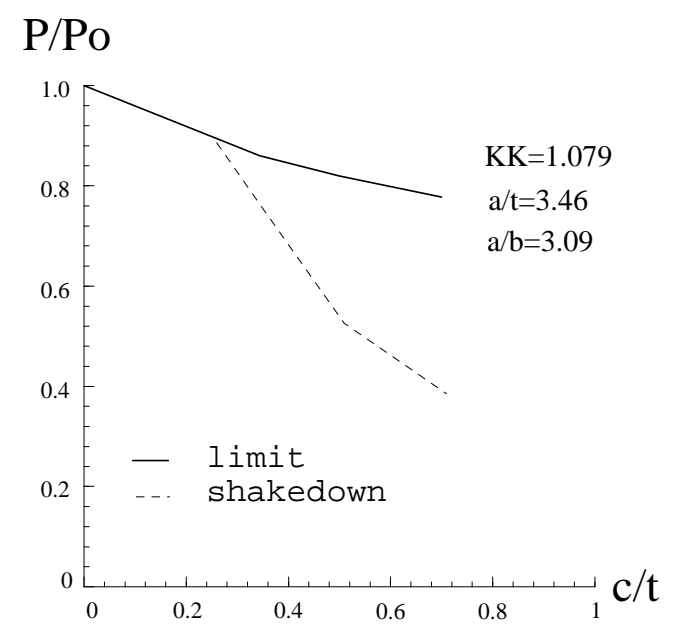

Fig. (5). Effect of depth of type B part-through pit on the carrying capacity.

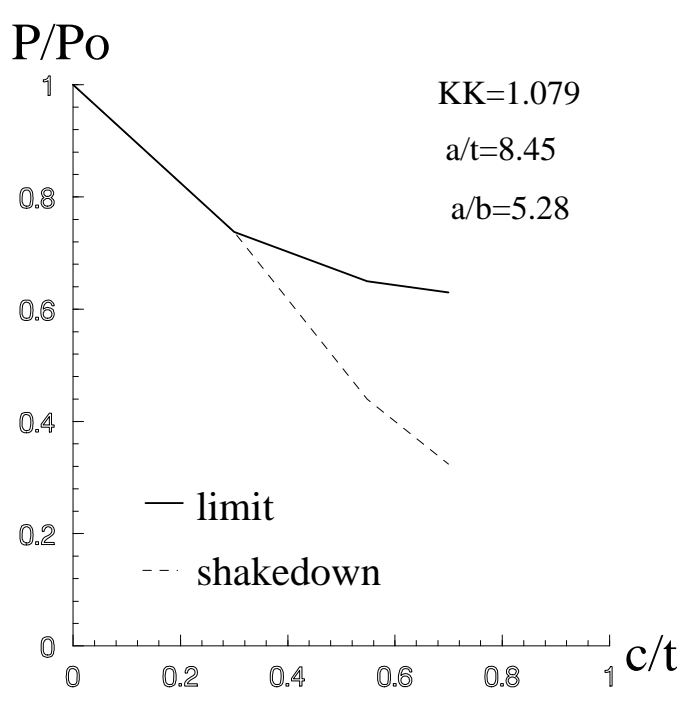

Fig. (6). Effect of depth of type C part-through pit on the carrying capacity.

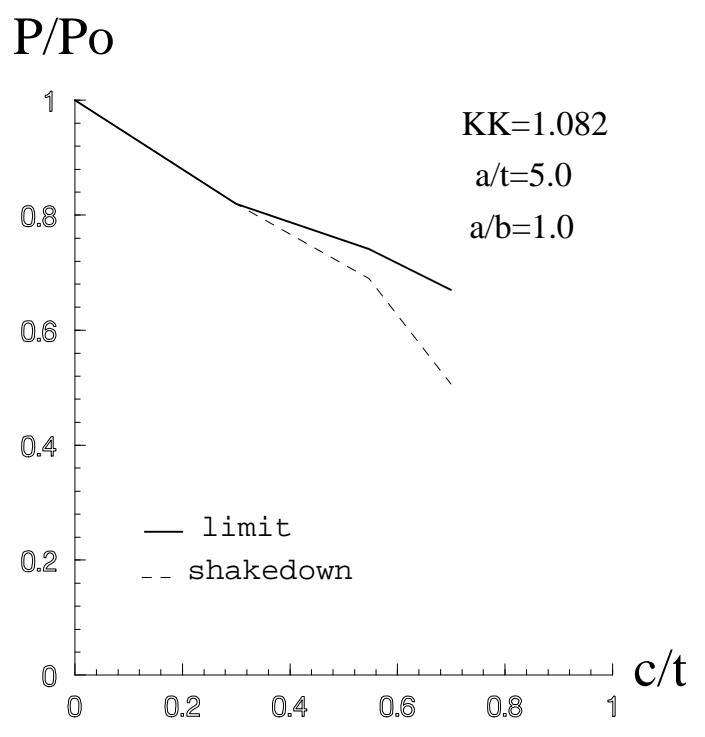

Fig. (7). Effect of depth of type D part-through pit on the carrying capacity.

The diagrams show that: (1) The carrying capacity of the tube with rectangular defect (its long axis is in the 
longitudinal direction of the cylinder) is the lowest and that with spherical one is the highest. (2) The carrying capacity of the tubes with rectangular or ellipsoidal defect decrease with their b/a. (3) The carrying capacity goes down with the expansion of the area of the pit, no matter what kinds of the pit is. (4) For the same model the shakedown pressure are coincident with limit one when the pit is shallow, but it descends more rapidly than limit one when the pit is deepened.

\section{CONCLUSIONS}

A FEM computational form based on the static shakedown theorem was developed in this paper. The circular tubes containing external pit defects were analyzed using Temperature Parameter Method, in which the computational quantity was largely decreased by simulating the residual stress field with a pseudo self-equilibrium thermal stress field. The approach was verified by the experimental results of six models. Due to the intrinsic characteristics of displacement element, the computational results should be greater than the theoretic lower-bound values, which are more closed to the real values. From the numerical results for the circular tubes with four types of part-though pits, it is found that rectangular defect is the most danger defect. The carrying capacity of the tubes decreases monotonously with the expansion of the pit. When a pit is shallow, the shakedown pressure of the tube is coincident with the limit one, however with deepening the pit, the two load carrying capacities becomes bifurcated, the shakedown pressure descends more rapidly than the limit one.

\section{CONFLICT OF INTEREST}

None declared.

\section{ACKNOWLEDGEMENTS}

The authors gratefully acknowledge the financial support provided by the Natural Science Foundation for the National Science Fund for Distinguished Young Scholars (No. 50925829), Natural Science Foundation for the team project of Guangdong Province (9351806001000001), the National Natural Science Foundation of China (No. 51078238), the Science and Technology Foundation of Shenzhen City (JC201005280571A) and the Science and Technology Foundation of Shenzhen University (No. 000015).

\section{REFERENCES}

[1] I. Milne, R.A. Ainsworth, A.R. Dowling, and A.T. Stewart, Assessment of the Integrity of Structures Containing Defects, R/H/R6-Rev.3, CEGB Report, UK, 1986.

[2] R. Kitching, and K. Zarrabi, "Lower bound to limit pressure for cylindrical shell with part-through slot", Int. J. Mech. Sci., vol. 23, pp. 31-48, 1981 .

[3] R. Kitching, and K. Zarrabi, "Limit and burst pressures for cylindrical shells with part-through slots", Int. J. Press. Vessels Piping, vol. 10, pp. 235-270, 1982.

[4] A.G. Miller, "Review of limit loads of structures containing defects", Int. J. Press. Vessels Piping, vol. 32, pp. 197-327, 1988.

[5] E. Melan, "Theoric statisch unbestimmter tragwerke aus idealplastischem baustoff', Sitzungsbericht der Akademie der Wissenschaften, Abt.IIA 145, Wien, p. 195, 1938.

[6] W.T. Koiter, "General theorems for elastic-plastic solids", In: Progress in Solid Mechanics I.N. Sneddon and R. Hill, Eds. Amsterdam: North-Hollad 1960, pp. 165-221.

[7] J.A. König, Shakedown of Elastic-Plastic Structures, PWN, Warsaw, Poland, 1987.

[8] X. Feng, and D. Gross, "A global/local shakedown analysis method of elastoplastic cracked structures". Eng. Fract. Mech., vol. 63, pp. 179-192, 1999.

[9] X.Q. Feng, and X.S. Liu, "On shakedown of three-dimensional elastoplastic strain-hardening structures”. Int. J. Plasticity, vol. 12, pp. 1241-1256, 1997.

[10] X.Q. Feng, and S.W. Yu, "Damage and shakedown analysis of dtructures with strainhardening”. Int. J. Plasticity, vol. 11, pp. 237 249, 1995.

[11] Y.H. Liu, Z.Z. Cen, and B.Y. Xu, "A numerical method for plastic limit analysis of 3-D structures", Int. J. Solids Struct., vol. 32, pp. 1645-1658, 1995.

[12] Y.H. Liu, Z.Z. Cen, and B.Y. Xu, "Numerical limit analysis of cylindrical shells with part-through slots", Int. J. Press. Vessels Piping, vol. 64, pp. 73-82, 1995.

[13] J.A. König, "On exactness of the kinematical approach in the structural shakedown and limit analysis", Arch. Appl. Mech., vol. 52, Number 6, pp. 421-428, 2004.

[14] P. Fuschi, and C. Polizzotto, "The shakedown load boundary of an elastic-perfectly plastic structure", Meccanica, vol. 30(2), pp. 155$174,2005$.

[15] F. Giambanco, L. Palizzolo, and L. Cirone, "Computational methods for optimal shakedown design of FE structures", Struct. Optim., Vol. 5, Numbers 3-4, pp. 284-295, 1998.

[16] C. Polizzotto, "A unified approach to quasi-static shakedown problems for elastic-plastic solids with piecewise linear yield surface", Meccanica, vol. 13, Number 2, pp. 109-120, 2005.

[17] M.D. Xue, X.F. Wang, F.W. Williams, and B.Y. Xu," Lowerbound shakedown analysis of axi-symmetric structures subjected to variable mechanical and thermal loads", Int. J. Mech. Sci., vol. 39, pp. 965-976, 1997.

[18] M.D. Xue, X.F. Wang, and B.Y. Xu. "Lower-bound shakedown analysis of spherical shells containing defects", Int. J. Press. Vessels Piping, vol. 68, pp. 287-292, 1996.

[19] K.J. Bathe, and E.L. Wilson, Numerical methods in finite element analysis, Prentice-Hall, Inc: Englewood Cliffs, New Jersey, 1976.

[20] J.C. Gerdeen, A critical evaluation of plastic behavior data and a united definition of plastic loads for pressure components, WRC Bul., No. 254, 1979, pp. 1-64. 